\title{
Avaliação da qualidade de vida no climatério
}

\section{Evaluation of quality of life in climacterium}

O estudo "Avaliação da qualidade de vida de mulheres no climatério atendidas em hospital-escola na cidade do Recife, Brasil" conduzido por Silva Filho e Costa1 e publicado neste fascículo da Revista Brasileira de Ginecologia e Obstetrícia (RBGO) aborda tema atual e emergente no campo da Ginecologia e mesmo da Saúde Pública. Foram entrevistadas 234 mulheres climatéricas usuárias de um serviço universitário da Região Nordeste do país com o intuito de avaliar a sua qualidade de vida, além da sua sintomatologia climatérica, por meio de instrumentos reconhecidos e validados no Brasil. Trata-se de um estudo transversal, descritivo, cujos resultados mostraram uma tendência à deterioração da qualidade de vida entre a população pesquisada, sendo esta aparentemente influenciada tanto por fatores biológicos relacionados ao hipoestrogenismo, como por fatores psicossociais.

A questão da qualidade de vida no climatério, a despeito da sua importância na atualidade, infelizmente ainda é pouco explorada no Brasil. A maioria dos estudos disponíveis tem sido realizada em outros países, não sendo sempre possível a transposição dos seus resultados para a realidade brasileira, em razão de diferenças culturais e socioeconômicas. Além disso, as poucas pesquisas realizadas no país incluem populações restritas a serviços de saúde isolados, o que dificulta a extrapolação de seus resultados para a população em geral ${ }^{1,2}$.

Como exemplo de estudo brasileiro abordando a questão da qualidade de vida no climatério está a pesquisa realizada na Universidade de Caxias do Sul (UCS), visando avaliar a qualidade de vida de 323 mulheres pós-menopáusicas com idade entre 45 e 60 anos. O instrumento utilizado foi o Women's Health Questionnaire (WHQ, Questionário da Saúde da Mulher). A sua análise revelou uma tendência à deterioração da qualidade de vida entre a população estudada, em particular nos domínios relacionados a sintomas somáticos, humor deprimido e ansiedade. Através de análise multivariada, observou-se que quanto menor a escolaridade e a frequiência da atividade sexual ( $\mathrm{p}<0,01)$, assim como a confirmação de co-morbidades clínicas prévias $(\mathrm{p}=0,03)$, piores os índices de qualidade de vida. Em contrapartida, a atividade física regular se associou a melhor qualidade de vida $(\mathrm{p}=0,01)$. A terapia hormonal, em especial, não se associou à qualidade de vida ${ }^{1}$. Achados similares foram obtidos por outro estudo, este realizado na Universidade Estadual de Campinas (Unicamp), ao comparar a qualidade de vida entre mulheres usuárias ( $n=106)$ e não usuárias $(n=101)$ de terapia hormonal. Os achados do estudo de Campinas não mostraram diferenças significativas entre os grupos avaliados, ainda que as usuárias de terapia hormonal tivessem apresentados menos sintomas climatéricos ${ }^{3}$. 
Outra pesquisa, esta conduzida por Favarato e Aldrighi, avaliou através de um estudo caso-controle o impacto da doença arterial coronariana (DAC) em grupo de $100 \mathrm{mu}-$ lheres pós-menopáusicas usuárias do Instituto do Coração (InCor) do HC-FMUSP. A análise de seus achados mostrou que a DAC contribuía para uma maior deterioração da qualidade de vida nos anos que se seguem a menopausa, limitando a sua capacidade física e o desempenho das atividades da vida diária, bem como intensificando as dificuldades emocionais comuns nessa fase ${ }^{4}$.

Em relação ao trabalho desenvolvido pelos colegas de Recife, seus resultados mostram-se também interessantes. Ainda assim, alguns comentários são necessários devido à metodologia adotada - em especial, o seu caráter descritivo, que restringiu a realização de análises mais aprofundadas dos dados obtidos, alguns muito interessantes e instigantes, principalmente ao levantar a hipótese da influência de fatores psicossociais na qualidade de vida no climatério, o que vai de encontro com o relatado por outros autores tanto em nível nacional, como internacional ${ }^{1,2,4,5-9}$.

O uso de modelos multivariados, ao permitir o controle de eventuais variáveis de confundimento, teria contribuído para um maior aprofundamento das questões levantadas, trazendo à tona possíveis fatores que estariam interferindo na qualidade de vida entre a população pesquisada. Em particular, a influência do estado menopausal na qualidade de vida e na sintomatologia climatérica poderia ter sido mais explorada. Tais comentários não devem ser vistos como críticas isoladas, mas como sugestões para futuros estudos ou mesmo uma nova análise dos dados apresentados neste periódico, com vistas a novas publicações com outros enfoques ${ }^{1,4,7,9}$.

A preocupação com a qualidade de vida em saúde ao longo do processo de envelhecimento tem aumentado exponencialmente nos últimos anos. Em parte, isso se deveu ao progressivo aumento da expectativa de vida ocorrido nas últimas décadas e ao reconhecimento pelos profissionais de saúde da importância dos sentimentos e percepções dos pacientes sobre a sua doença, assim como da monitorização do seu bem-estar frente a medidas terapêuticas voltadas a prolongar a sua vida, aliviar a dor, restaurar funções e prevenir incapacidades ${ }^{2}$.

No que tange ao climatério, vale lembrar a maior expectativa de vida da mulher brasileira, que ultrapassa em cerca de dez anos a masculina. As mulheres com mais de 40 anos já correspondem a 32\% do contingente populacional do Brasil, devendo sofrer um aumento de $11 \%$ até $2010^{7,9}$. Conseqüentemente, espera-se que nos próximos anos ocorra uma procura crescente nos serviços do país por mulheres com queixas relacionadas ao climatério, trazendo uma maior preocupação com a saúde e qualidade de vida desta parcela da população ${ }^{1,2,10}$.

Além das questões demográficas, parte da preocupação atual com as questões relacionadas à qualidade de vida no climatério deveu-se à mudança nos paradigmas da assistência prestada a essa etapa da vida feminina ocorrida nos últimos anos, principalmente após a publicação do estudo Women's Health Initiative (WHI). Ainda que seus resultados tenham sido posteriormente questionados por problemas metodológicos e de amostragem, o WHI, ao mostrar uma associação entre o uso de terapia estroprogestativa contínua e a ocorrência de câncer de mama e eventos cardiovasculares, fomentou uma revisão da assistência à mulher no climatério, que passou a ter como eixo norteador a qualidade de vida ${ }^{2,11,12}$.

Atualmente, a qualidade de vida é reconhecida como um indicador de eficácia, eficiência e impacto de eventuais intervenções voltadas à prevenção ou tratamento de agravos à saúde, tanto individuais como em nível populacional. Na sua avaliação, dois aspectos são fundamentais, a subjetividade e a multidimensionalidade. A primeira refere-se à percepção do próprio indivíduo acerca do seu estado de saúde e dos aspectos não-médicos relativos ao seu contexto de vida, ou seja, cada indivíduo avalia a sua situação em cada uma das dimensões relacionadas à qualidade de vida, o que não pode ser feito por um observador externo. A multidimensionalidade, por sua vez, decorre do reconhecimento da multiplicidade de fatores envolvidos na qualidade de vida de um indivíduo ${ }^{13}$.

Quanto à definição da expressão "qualidade de vida", duas tendências emergem: a qualidade de vida como um conceito mais genérico e a qualidade de vida relacionada à saúde (health-related quality of life). A primeira envolve uma concepção mais ampla de qualidade de vida, aparentemente influenciada pela Sociologia, indo ao encontro da definição adotada pela Organização Mundial da Saúde (OMS), segundo a qual qualidade de vida refere-se à percepção do indivíduo sobre a sua posição na vida, no contexto da cultura e dos sistemas de valores em que vive, considerando-se seus objetivos, expectativas e preocupações pessoais ${ }^{1,2,13,14}$. Mais recentemente, outros autores têm destacado a influência de fatores físicos, mentais, psicológicos, sociais, econômicos, culturais e espirituais, além da saúde física na qualidade de vida ${ }^{15}$. Já hoje se reconhece qualidade de vida como um construto multidimensional, com significados diferenciados segundo a diversidade de contextos de vida. Entre as dimensões que lhe dão significado, estão a manutenção da capacidade funcional, a satisfação pessoal, o estado emocional e a interação social ${ }^{1,2}$.

Os estudos que partem de uma definição mais genérica de qualidade de vida têm por característica permitir a inclusão de indivíduos saudáveis ou não, não se restringindo aos portadores de agravos específicos. Como exemplo de instrumentos genéricos de avaliação da qualidade de vida está o World Health Organization Quality of Life Assessment (WHOQOL), nas suas diferentes versões, e o MOS SF-36 Health Survey ${ }^{13-15}$. 
Já a qualidade de vida relacionada à saúde, além de aspectos gerais, contempla aspectos relacionados a enfermidades específicas ou a eventuais intervenções em saúde. Porém, a sua avaliação tem mostrado algumas divergências. Alguns autores defendem enfoques mais específicos da qualidade de vida à medida que esses favorecem a identificação das características específicas de um determinado agravo. Outros, por sua vez, apontam que algumas medidas de qualidade de vida relacionada à saúde têm abordagens excessivamente restritas a sintomas ou disfunções, dificultando uma percepção mais ampla dos aspectos não médicos relacionados à qualidade de vida ${ }^{13,14}$.

Tentando contornar as divergências acima, os instrumentos de mensuração da qualidade de vida em saúde têm sido elaborados de forma a manter o caráter multidimensional, para que permitam avaliar também a percepção geral de qualidade de vida, mesmo que o seu foco recaia sobre os sintomas, incapacidades ou limitações decorrentes de determinada enfermidade ${ }^{13,14}$. No climatério, alguns dos instrumentos que avaliam a qualidade de vida em saúde são o WHQ, o Menopause Rating Scale (MRS) e o Menopause-Specific Quality of Life Questionnaire (MENQOL), entre outros ${ }^{15}$.

Como lembrado por Zahar et al. ${ }^{16}$ e seguido por Silva Filho e $\operatorname{Costa}^{1}$, no estudo apresentado neste fascículo, na avaliação da qualidade de vida no climatério é recomendável a combinação de instrumentos genéricos e específicos por potencializar a qualidade dos resultados obtidos. Os instrumentos específicos contribuem para um maior aprofundamento do impacto dos sintomas menopáusicos e a sua relação com a qualidade de vida, enquanto que os instrumentos genéricos permitem ampliar o conhecimento das mudanças físicas e mentais que influenciam a qualidade de vida no climatério, não se limitando aos aspectos particulares dessa etapa da vida feminina ${ }^{15}$.

Mais recentemente, tem surgido a proposta de utilizar também métodos qualitativos nas pesquisas voltadas a avaliar a qualidade de vida no climatério, a despeito de a metodologia quantitativa ainda se mostrar hegemônica e predominante na literatura especializada, principalmente entre a classe médica. A metodologia qualitativa, quando adequadamente conduzida, permite que se mostrem com mais liberdade a subjetividade e a multidimensionalidade - tão importantes na aferição da qualidade de vida. Além disso, os defensores de enfoques qualitativos enfatizam que a utilização de medidas padronizadas pode levar a respostas estereotipadas, que muitas vezes têm pouco ou nenhum significado para a pessoa ${ }^{16,17}$.

No estudo da qualidade de vida, alguns aspectos devem ser considerados. Cerca de 50 a 70\% das mulheres vêm a referir sintomas somáticos e dificuldades emocionais no climatério, interferindo no seu bem-estar. São também comuns, nessa fase, queixas relacionadas a dificuldades com o sono, perda da libido, cefaléia, mialgias e artralgias, maior ansiedade e até depressão. Todavia, persistem controvérsias sobre o quanto tais queixas são decorrentes somente da queda estrogênica ou de fatores psicossociais relacionados ao próprio processo de envelhecimento. As ondas de calor e a atrofia urogenital seriam as únicas queixas diretamente relacionadas à queda dos níveis estrogênicos. As demais estariam relacionadas principalmente à dificuldade de aceitar o processo de envelhecimento - este simbolizado pela menopausa ${ }^{1,2,18}$.

Do ponto de vista antropológico, vale lembrar que, nas culturas ocidentais, a beleza física, a juventude e a maternidade são elementos de valorização feminina, cuja perda pode favorecer sentimentos de desvalia, tristeza e até depressão. Além disso, o climatério coincide freqüentemente com a independência dos filhos, a aposentadoria e a perda de familiares, situações estas que demandam adaptações emocionais nem sempre fáceis para a mulher ${ }^{1,2}$.

Segundo Hunter ${ }^{9}$, dentre os fatores associados à qualidade de vida da mulher ao longo do seu processo de envelhecimento, os mais relevantes são as suas condições físicas e emocionais prévias, a sua inserção social e experiências frente a eventos vitais. Mais recentemente ${ }^{17-19}$, verificouse que a sintomatologia climatérica e a qualidade de vida no climatério eram influenciadas também pelas atitudes e percepções das próprias mulheres em relação à menopausa, o que pode ter ocorrido no estudo de Silva-Filho e Costa ${ }^{1}$.

Como se pode observar, o tema "qualidade de vida”, em especial no climatério, pode ser visto pelos mais diversos olhares, seja da ciência, por meio de várias disciplinas, seja do senso comum, seja do ponto de vista objetivo ou subjetivo, seja em abordagens individuais ou coletivas. No âmbito da saúde, quando visto no sentido ampliado, ele se apóia na compreensão das necessidades humanas fundamentais, materiais e espirituais e tem seu foco mais relevante no conceito de promoção da saúde ${ }^{14}$.

Quando vista de forma mais focalizada, qualidade de vida em saúde coloca sua centralidade na capacidade de viver sem doenças ou de superar as dificuldades dos estados ou condições de morbidade. Isso porque, em geral, os profissionais atuam no âmbito em que podem influenciar diretamente, isto é, aliviando a dor, o malestar e as doenças, intervindo sobre os agravos que podem gerar dependências e desconfortos, seja para evitá-los, seja minorando conseqüências dos mesmos ou das intervenções realizadas para diagnosticá-los ou trata-los ${ }^{14}$. Nesse contexto, a preocupação com a qualidade de vida se contrapõe com a tendência moderna de tecnologização excessiva da Medicina, contribuindo para que se resguarde um dos mais atuais paradigmas da saúde, a questão da humanização, o que vai de encontro, inclusive, com o proposto pelos inúmeros protocolos e consensos em relação ao climatério nessa era pós-WHI. 


\section{Referências}

1. Silva-Filho EA, Costa AM. Avaliação da qualidade de vida de mulheres no climatério atendidas em hospital-escola na cidade do Recife, Brasil. Rev Bras Ginecol Obstet. 2008;30(3): 1 13-20.

2. De Lorenzi DRS, Baracat EC, Saciloto B, Padilha Júnior I. Fatores associados à qualidade de vida após menopausa. Rev Assoc Med Bras. 2006;52(5):312-7.

3. De Lorenzi DRS, Baracat EC. Climatério e qualidade de vida. Femina. 2005;33(12):899-903.

4. Favarato MECS, Aldrighi JM. A mulher coronariopata no climatério após a menopausa: implicações na qualidade de vida. Rev Assoc Med Bras. 2001;47(4):339-45.

5. Zahar SEV, Aldrighi JM, Pinto Neto AM, Conde DM, Zahar LO, Russomano F. Qualidade de vida em usuárias e não usuárias de terapia de reposição hormonal. Rev Assoc Med Bras. 2005;51(3):133-8.

6. Dennerstein L, Lehert P, Guthrie J. The effects of the menopausal transition and biopsychosocial factors on well-being. Arch Womens Mental Health. 2002;5(1):15-22.

7. Gonçalves R, Merighi MAB, Aldrighi JM. Reflexões sobre o climatério com enfoque no corpo, na cultura e na subjetividade. Reprod Clim. 2003; 18:108-12.

8. Olofsson AS, Collins A. Psychosocial factors, attitude to menopause and symptoms in Swedish perimenopausal women. Climacteric. 2000;3(1):33-42.

9. Hunter MS. Predictors of menopausal symptoms: psychosocial aspects. Baillieres Clin Endocrinol Metab. 1993;7(1):33-45.

10. Schwarz S, Völzke H, Alte D, Schwahn C, Grabe HJ, Hoffmann $\mathrm{W}$, et al. Menopause and determinants of quality of life in women at midlife and beyond: the Study of Health in Pomerania (SHIP). Menopause. 2007;14(1):123-34 .

11. Pedro AO, Pinto-Neto AM, Costa-Paiva L, Osis M, Hardy E. Procura de serviço médico por mulheres climatéricas brasileiras. Rev Saúde Pública. 2003;36(4):484-90.

12. Rossouw JE, Anderson GL, Prentice RL, LaCroix AZ, Kooperberg C, Stefanick ML, et al. Risks and benefits of estrogen plus progestin in healthy postmenopausal women: principal results from Women's Health Initiative randomized controlled trial. JAMA. 2002;288(3):321-33.

13. Mendelsohn ME, Karas RH. HRT and the young at heart. N Engl J Med. 2007;356(25):2639-41.

14. Seidl EMF, Zannon CMLC. Qualidade de vida e saúde: aspectos conceituais e metodológicos. Cad Saúde Pública. 2004;20(2):580-8.

15. Minayo MCS, Hartz ZMA, Buss PM. Qualidade de vida e saúde: um debate necessário. Ciênc Saúde Coletiva. 2000;5(1):7-18.

16. Zahar SEV, Aldrighi JM, Tostes MA, Russonamo F, Zahar LO. Avaliação de qualidade de vida na menopausa. Reprod Clim. 2001;16(3):163-6.

17. Vigeta SMG, Brêtas ACP. A experiência da perimenopausa e pósmenopausa com mulheres que fazem uso ou não da terapia de reposição hormonal. Cad Saúde Pública. 2004;20(6):1682-9.

18. Lindh-Astrand L, Hoffmann M, Hammar M, Kjellgren KI. Women's conception of the menopausal transition-a qualitative study. J Clin Nurs. 2007; 16(3):509-17.

19. De Lorenzi DRS, Danelon C, Saciloto B, Padilha Júnior I. Fatores indicadores da sintomatologia climatérica. Rev Bras Ginecol Obstet. 2005;27(1):12-9. 